\title{
UN ENFOQUE A LA ORGANIZACIÓN ESCOLAR A PARTIR DE LA TEORÍA NEOCLÁSICA
}

\author{
Randolf Aguirre Aguirre
}

\author{
Recibido 18-VI-2001 • Aceptado 12-III-2002
}

\begin{abstract}
Resumen: El presente artículo tiene como propósito presentar un breve esbozo sobre la teoría neoclásica que se ha insertado en la gestión institucional, haciendo que los centros educativos aparezcan disfuncionales e incapaces de estimular el desarrollo de las facultades del ser humano, lo que ha hecho que la escuela en su función socializadora haya merecido las críticas tanto de intelectuales como de otros miembros de la sociedad, las cuales han hecho ver que es cada vez más necesario el cambio en las estructuras operacionales de estas entidades, requiriéndose de mayor flexibilidad y dinamismo en los procesos tanto técnicos como políticos, es por ello que se hace referencia al modelo de organizaciones inteligentes como una alternativa de desarrollo institucional.
\end{abstract}

\section{Introducción}

Presentar un análisis de la administración escolar es una tarea que está revestida de una gran complejidad, no solamente por tratarse de una actividad que influye en la conducta y el interés particular que pueden tener cada uno de los miembros de la organización, sino también, por momentos en que nuestra sociedad se encuentra inmersa en una coyuntura sin precedentes, en donde pareciera que la constante lucha por el poder, la masificación de los niveles de información, la conformación de modelos sociales cada vez más organizados (alianzas estratégicas, tratados de libre comercio y la globalización de los mercados), y por último, la incorporación de nuevas tecnologías en los medios de producción que han traído como consecuencia el aumento en la generación de las mercancías, reduce la capacidad de empleo; que en resumen es producto del desarrollo beligerante que experimenta en nuestros días el capitalismo, aumentando con ello la desigualdad social. Todo este acontecer social hace que el enfoque tradicional de la administración educativa pierda actualidad y lucidez; sin poder constituirse en una alternativa de desarrollo nacional, si no más bien, en un problema inconmesurable en la formación de las generaciones más jóvenes. 
Por lo tanto, el presente artículo tiene como propósito presentar un breve esbozo sobre la teoría neoclásica que se ha insertado en la gestión institucional, haciendo que los centros educativos aparezcan disfuncionales e incapaces de estimular el desarrollo de las facultades del ser humano, lo que ha hecho que la escuela en su función socializadora haya merecido las críticas tanto de intelectuales como de otros miembros de la sociedad, las cuales han hecho ver que es cada vez más necesario el cambio en las estructuras operacionales de estas entidades, requiriéndose de mayor flexibilidad y dinamismo en los procesos tanto técnicos como políticos, es por ello que se hace referencia al modelo de organizaciones inteligentes como una alternativa de desarrollo institucional.

\section{Un enfoque a la organización escolar a partir de la teoría neoclásica}

La crisis del Estado costarricense no es solamente la consecuencia de las políticas económicas que afectan a la comunidad internacional, es también producto de la inapropiada organización que han venido experimentando las instituciones públicas, desde administraciones anteriores, provocando en la mayoría de los ciudadanos desconfianza, tanto para satisfacer sus necesidades individuales como para convertirse en efectivas alternativas en el desarrollo nacional.

En este sentido el desarrollo de alternativas ha estado marcado por esa realidad en donde las soluciones no pueden seguir siendo convencionales, es decir, que solamente se quedan en planes y proyectos con buenas intenciones, pero que más tarde se vuelven frustrantes.

Uno de los dilemas a los que se ve confrontado este campo es precisamente sobre el tema de la calidad, el cual no ha estado lo suficientemente claro para ser aplicado al desarrollo de la educación, pues el término se ha tenido como un eslobismo más, que decora la elocuencia de los discursos políticos, en los cuales se ha afirmado que lo importante de pretender el mejoramiento permanente de la enseñanza está en la agregación de días en el horario escolar, la implementación de materiales didácticos, y la dotación de tecnología de punta a los centros educativos, pero en realidad el concepto tiene múltiples acepciones, por lo tanto, toca inexorablemente a todos los elementos y factores que confluyen en esta actividad.

Esta claridad que no se ha tenido en las esferas de la administración educativa permitió que se comprendiera el término como sinónimo de productividad, señalándose como índices de esta tarea las promociones escolares. Al comprenderse así la calidad se concibió a los estudiantes como insumos que después de un largo proceso podrían ser incorporados a la sociedad, puesto que ya se les había dotado, presuntamente, de conocimientos, habilidades y destrezas que podrían ser puestas en práctica no solo para su desarrollo individual, si no también de su comunidad. Esta visión utilitarista trajo como consecuencia que nuestros graduados se percibieran como un producto más en el mercado, que podría incorporarse a las actividades económicas del país. No obstante, las deficiencias en el aprendizaje han hecho que muchos de ellos puedan incorporarse como obreros en las industrias, las fábricas, el comercio y en algunos otros sectores de la economía informal. De este modo la aspiración vocacional del educando queda relegada a un segundo plano, teniendo que conformarse con la ocupación de un puesto que dista mucho de su talento y capacidades.

Vista así la educación ésta aparece en los albores del siglo XXI en crisis, pues solamente ha podido promover un aprendizaje dentro de sus estructuras, el cual ha favorecido un sistema capitalista y democrático, que ha sostenido y perpetuado a una élite en el poder. Desde luego ésta ha sido una realidad que, no solamente, ha 
estado presente en nuestro país, sino también, en el resto de los países latinoamericanos, esto ha insentivado a muchos profesionales de las ciencias sociales, no solamente a investigar más sobre el estado de decadencia que ha experimentado la educación en nuestros días y como producto de su quehacer intelectual se ha responsabilizado, a la adopción de esquemas operativos que no son compatibles con esta actividad, sino también como ya se dijo la influencia de factores externos.

Es así como Ivan Illich (1979), explica que la escuela conjuntamente con todas sus estructuras se ha convertido en un monopolio del conocimiento considerándose que solo ella es capaz de enseñar, excluyendo de toda participación a otros actores sociales. Ante esta situación Illich propone una escuela sin paredes, en donde la actividad pedagógica sea entregada a un grupo de personas selectas de la sociedad.

Por su parte Pablo Freire (1987), demanda de la institución escolar un cambio radical, proponiendo lo que él ha denominado la pedagogía crítica, que ante todo es una visión que se asienta en el respeto por la vida, la esperanza y la visión de futuro, que inspira sin pretender en ninguna manera consolar a los oprimidos, si no más bien promover formas duraderas de crítica y lucha contra las fuerzas objetivas, opresivas al combinar la dinámica de la crítica y la lucha colectiva con una filosofía de la esperanza. Freyre ha creado un lenguaje de posibilidades a lo que él llama una visión profética permanente y bajo esta visión que esconde una adhesión genuina hacia una esperanza que presupone nuestra capacidad para la lucha. En este mismo sentido Horacio Radetich (1983), es conciente que las clases de conocimiento existentes están comprometidas con un sistema ideológico determinado. Para Radetich el saber del capitalismo es la aprehensión de las habilidades requeridas para su desarrollo, y por lo tanto, la reducción de las capacidades de aprendizaje a las condiciones del capitalismo.
Puede interpretarse que ésta es tan solo una de las críticas que algunos ideólogos marxistas en una forma analítica y concreta han hecho a la educación que se desarrolla dentro de los esquemas dominados por los medios de producción que pertenecen a la burguesía capitalista, no obstante esto implica el hecho de no reconocer la urgencia de un cambio organizacional que requieren los centros escolares, ya que autores como Leonor Meza Spitta (1999) ha sostenido que aún se encuentran presentes en los sistemas educativos de la región, están presentes algunos rasgos del pensamiento de la escuela clásica, puesto que éstas tendencias provocaron en la administración educativa: una división del trabajo en autoridad, responsabilidad, unidad de mando, disciplina, jerarquización, centralización y orden. Por otra parte, Michael Crozier (1992), ha advertido que la crisis que enfrentan los servicios públicos administrados por el Estado con sentido social pasa por considerar la falta de respuesta que las entidades experimentan no solamente ante la falta de recursos económicos, bienes y servicios, si no también se carece de un planteamiento organizacional que haría más eficaz la vida operacional de estas instituciones. A esta realidad desde luego no escapan nuestras instituciones dedicadas a la enseñanza, en donde su incapacidad de respuesta ante los dilemas que plantean las diversas coyunturas sociales se ha responsabilizado al Estado por su marcada centralización en la administración de este servicio público.

No obstante, pese a ello, todo centro escolar es capaz de gestar su propio comportamiento organizacional, que está influido por principios que legitiman su accionar y definen su cultura organizacional. Para Santos Guerra (1992), ésta tiene tres características fundamentales en las que puede darse, a lo que definitivamente muchos miembros aceptan teórica y prácticamente, sin prestar resistencia. Entre estos rasgos están los siguientes: 
- La cultura organizacional de la escuela es individualista dado que cada profesor actúa en su aula y cada alumno es responsable de su aprendizaje.

- La cultura de la escuela es de rutinas puesto que cada día se entra a la misma hora, se construyen los horarios de la misma forma, se disfrutan los recreos durante el mismo tiempo.

- La cultura de la escuela es una cultura de rendimientos, puesto que se valoran más los resultados que los esfuerzos.

De conformidad con las características que reviste el comportamiento organizacional prevaleciente en los centros educativos, su organización puede ser abordada desde la perspectiva de un enfoque neoclásico, en tanto que el tratamiento de los problemas de la productividad, estén dados por los principios que rigen en forma absoluta como son en la escuela: la programación y logros de los objetivos, la aplicación de pruebas estandarizadas en las que se mide por igual el rendimiento de los alumnos, los altos índices de promoción escolar que se han concebido como sinónimos de calidad (eficiencia en la productividad), y el hecho de percibir a los agentes educativos como insumos que son objeto de una transformación gradual a través de acciones controladas. Todo ello forma parte del clima organizacional que afecta tanto el desempeño como la productividad de todos aquellos que de alguna manera se encuentran inmersos en el proceso educativo.

También podemos agregar que la estructura metodológica que impulsaron los precursores de la escuela neoclásica ha hecho la enunciación de siete principios para hacer más eficiente la vida operacional de una organización, de los cuales cuatro se encuentran implícitos también en nuestros centros educativos y son los siguientes:

\section{Unidad de mando}

Este principio Chiavenato (1992), lo explica señalando que para toda actividad productiva es necesaria la dirección de un jefe para organizar los trabajos de los subordinados, puesto que estos siempre requieren saber quiénes son sus superiores. En la organización escolar existe una cultura jerarquizada en donde los supervisores mandan al director, el director manda al profesor y por último el profesor manda a sus estudiantes.

\section{Nivel de especialización}

La aplicación de este principio para los neoclásicos les permite aumentar la eficiencia distinguiéndose en este sentido dos tipos de especialización, entre los que se encuentran: tipo de actividad y tipo de proceso. Encontramos cierto nivel de correlación en la estructura funcional del sistema educativo, por ejemplo, los procesos de planificación administrativa son siempre diferentes a la planificación pedagógica que llevan a cabo los docentes, lo que a nuestro criterio ambas deben considerarse complementarias una de la otra, puesto que se trata de una propuesta colegiada, que se hace efectiva en las aulas de conformidad con las necesidades que presentan los estudiantes.

Por otra parte, se ha tenido el trabajo por áreas en la enseñanza primaria, que tiende a especializar el trabajo de los profesionales de la educación, en tanto se ocupan de dar clases de una sola materia para alcanzar la eficiencia en el proceso educativo. Cada quién se constituye en un verdadero inspector de calidad de su trabajo sin que se puedan admitir tanto experiencias como estilos pedagógicos de otros colegas. Un ejemplo claro de esta dinámica son las aulas integradas que pareciera haberse convertido en los últimos años en "escuelas unidocentes" en donde el conocimiento 
formal y la planificación de todas las experiencias de aprendizaje que se puedan generar en estos recintos son de la absoluta responsabilidad del profesional de la educación especial y, esta ha sido la tónica a la inversa en donde los docentes regulares han cuestionado en los últimos años lo inconveniente de tener estudiantes con discapacidad por no tener la preparación necesaria que les permita desempeñar apropiadamente dicha labor. Este tipo de criterios fue lo que tuvo a esta población durante muchos años relegada sin ninguna posibilidad de interactuar con sus semejantes.

Está claro que los niveles de especialización no solamente se presentan en la escuela, si no más bien, se encuentran presentes en todo el Sistema Educativo. El elevado crecimiento que en los últimos años ha experimentado el Ministerio de Educación Pública, tanto por la expansión de servicios provenientes de una demanda social de más y mejor educación, como por las nuevas corrientes educativas (tecnología educativa, televisión educativa, sistemas abiertos de educación) ha obligado al Ministerio a estructurarse haciendo que organizacionalmente dependa cada vez más de niveles de especialización para cumplir con el cometido que la sociedad le ha impuesto.

\section{Autoridad y responsabilidad}

De conformidad con Eduardo Kastica (1992), expresa que para los precursores de esta teoría, este principio está dado en la capacidad de llevar a la práctica la autoridad mediante la delegación de responsabilidades. Para este caso la organización escolar en el consejo de profesores a través de su director delega responsabilidades mediante la formación de comités que se encargan de actividades culturales, sociales, deportivas y evaluativas, entre otras. Así mismo, la creación en los últimos 25 años de direcciones regionales y circuitos escolares refleja claramente la idea de concentrar ciertas funciones del Ministerio de Educación en organismos inferiores que desarrollan tareas de supervisión y coordinación de los procesos de enseñanza y aprendizaje.

\section{Autoridad de línea y Estado Mayor}

La enunciación de este principio surge a partir del crecimiento de las labores de una organización, dado que es cada vez más necesario que un grupo de expertos o especialistas brinden a los altos mandos un modelo de asesoría que permita coordinar las tareas de los departamentos.

En la nomenclatura que presenta el Sistema Educativo se observa que sus entidades están integradas por diferentes departamentos que corresponden a cada materia, los cuales están a cargo de un coordinador especialista en esta materia y tiene la responsabilidad de coordinar y presentar los informes para cada ciclo lectivo.

Por otra parte, la autoridad de línea es entendida por esta escuela de pensamiento administrativo como aquella capacidad gerencial que se tiene para ejercer el dominio y el control de los grupos socialmente subordinados en toda relación productiva; por tanto, se puede notar con suma frecuencia cómo las prácticas que se dan en la administración de los servicios educativos están cada vez más orientadas a ordenar los grupos escolares, de tal forma que sean manejables por el docente y, en algunos casos se ha llegado a señalar por los auditores del Ministerio de Educación que los grupos superiores a los 35 estudiantes pone en riesgo el logro de los objetivos del programa escolar al perder el maestro el control de la disciplina y la estabilidad del grupo.

En general diremos que la imposibilidad que han experimentado nuestros centros educativos para satisfacer las espectativas y demandas del contexto, se ha 
debido esencialmente, como ya se explicó, a la existencia de rasgos que obedecen a leyes y principios que están dentro de las concepciones teóricas de la escuela neoclásica; es así como la administración escolar se percibe como un sistema cerrado, en donde quienes participan de sus funciones y papeles están fijados por las directrices que establece la gestión administrativa, la cual pretende un comportamiento organizacional normativo con vistas a alcanzar racionalmente los objetivos del programa. En este sentido el administrador neoclásico está atento al cumplimiento de las normas que rigen la organización educativa, por tanto, su preocupación consiste en crear un clima organizacional que favorezca la eficacia del Sistema Educativo para el logro de sus objetivos, relegando a un plano de importancia secundaria las interacciones que se puedan tener con el contexto social.

Son cada vez más urgentes cambios en los estilos de administración educativa que privilegien un mejor servicio educativo, siendo su razón de ser los estudiantes y sus necesidades, las cuales en su mayoría surgen de los avatares que plantean los factores económicos y sociales y, en este sentido no puede olvidarse que el administrador de la educación tiene en sus manos la tarea de orientar los recursos, esfuerzos y acciones necesarias para que las instituciones educativas realmente promuevan la formación integral de los individuos en una sociedad cambiante y cada vez mejor.

La apertura al cambio y una gestión administrativa integral pasa por considerar las necesidades y expectativas que tienen, estudiantes, docentes y administradores de la educación en un conjunto de situaciones dadas; sobre todo frente a las implicaciones que plantea el hecho de vivir en una sociedad post moderna que ha puesto sobre la palestra el cambio de las relaciones humanas.

La libertad del hombre de hoy está cada vez más comprometida y amenazada por los intereses que tiene una clase social, que es dueña de los medios de producción y que gusta como en el pasado de una visión utilitarista en la que el obrero, se concibe ante todo como un instrumento generador de riqueza. Es por lo tanto, cada vez más cierto que cada día el capitalismo se extiende como una ola expansiva que afecta a todos los estratos y modelos de socialización contemporáneos, a fin de tener un aumento en la mano de obra, de hombres y mujeres a los que se les ofrece un salario que en todo caso afecta su dignidad.

Tal y como está planteado el Sistema Educativo desde el primer al cuarto ciclo no propicia el desarrollo de facultades prevocacionales para el trabajo o elección de una carrera. Muchos ante la realidad de haber salido de la enseñanza secundaria, se encuentran desconcertados por lo que optan integrarse a los medios de producción fabril e industrial, y si ha esto se suma que todavía universidades que entre sus servicios ofrecen test vocacionales para ayudar a los estudiantes a encontrar su verdadera vocación, no podemos dudar que la educación tal y como está planteada solamente ha cumplido la tarea de transmitir conocimientos, que no son suficientes para alcanzar una remuneración que esté dentro de los estándares de la mano de obra calificada.

Con lo expuesto hasta aquí se hace necesario remosar el modelo de administración que se ha venido poniendo en práctica en nuestras instituciones que han estado dedicadas a la enseñanza mediante la adopción de aquellas decisiones referidas a la dinámica interna que tiene todo Centro Educativo, por tanto, deberán contemplarse las distintas dimensiones de la organización, dando a cada uno el lugar que le corresponde en la relación con los demás $\mathrm{y}$ atendiendo el contexto.

En esta misma tesitura Peter Senge (1992), ha ofrecido un nuevo paradigma organizacional en el cual las organizaciones sociales, más que ordenar, tendrán que discurrir sobre cómo pueden aprovechar el entusiasmo y la capacidad de las 
personas en todos los niveles de la organización. Desde el punto de vista metodológico los centros de enseñanza enfrentan el desafío de corregir sus errores, de aprender de la experiencia, tomar en consideración los requerimientos de un entorno cambiante, desarrollar y consolidar esquemas de comprensión y de acción y, en su orientación permanente hacia el logro de los fines, de las metas y los objetivos que le son propios.

En resumen, es posible decir que se convierten en sistemas adaptativos, puesto que para Senge la inteligencia de los organismos vivos es considerada en la actualidad como una propiedad emergente, en donde los componentes requieren de ciclos completos de aprendizaje y el desarrollo de procedimientos autocorrectivos que hagan posible la mejora continua de sus procesos y sus resultados.

El aprendizaje organizacional requiere de procedimientos sistemáticamente ordenados, así como de mecanismos para la corrección de errores, pero ante todo requiere de la voluntad de aprender que en nuestros días resulta equivalente a la voluntad de mejorar, todo ello no tendría ningún sentido si no se parte del compromiso previo que todos los miembros deben tener con la organización, pues se requiere que todos actúen sinérgicamente bajo leyes, principios, programas y proyectos intencionales que tiendan a formar y a su vez, correspondan a un desempeño eficaz de la institución.

La teoría de las organizaciones inteligentes aplicada a los centros dedicados a la enseñanza, debe comprenderse como las posiblidades que ofrece el medio para que sus miembros puedan expandir continuamente su actitud para lograr los resultados que desean, donde se cultivan nuevos patrones de pensamiento, siendo la aspiración pedagógica una verdadera propuesta colectiva. Tratándose en este sentido de que cada uno asuma la tarea de interpretar aquellos factores, tanto externos como internos que pueden ir en detrimento de la calidad de la educación y esto a su vez significa recrear el futuro de una institución educativa a partir de la experiencia y el aporte de todos sus miembros, sin que para ello se entre a considerar lo relevante del puesto. Todos merecen la posiblidad de ser escuchados en torno a temas que son siempre relevantes en toda organización, como son las relaciones humanas, el rendimiento escolar, la deserción, la programación escolar, y en general, la instauración de proyectos que tiendan a mejorar las relaciones del centro con el contexto social.

Para lograr el cambio de una organización tradicional a otra inteligente, Senge (1992), propone considerar cinco actitudes o disciplinas que deberá asumir la organización en general. Las disciplinas tratadas por Senge son las siguientes: Modelos mentales, Construcción de una visión compartida, Aprendizaje en equipo, Dominio personal y Pensamiento sistémico.

Veamos cada una de ellas brevemente en la forma en cómo pueden adaptarse al desarrollo operativo de entidades educativas siguiendo el sentido práctico y conceptual del autor:

\section{Los modelos mentales}

Estos son comprendidos como modelos mentales que están ondamente arraigados en la conciencia de los individuos. Se trata en general de imágenes y conceptos que influyen en la forma de cómo llegamos a comprender el mundo actual, sin que en general, nos percatemos de esta particularidad.

Se pone en evidencia que la aplicación de esta actitud en las escuelas equivale a romper con las viejas prácticas educativas que tanta frustración y desánimo han provocado en las generaciones más jóvenes. Implica adoptar cambios en todos los ámbitos que tiendan a dar libertad y disfrute a la experiencia de aprender dejando atrás las rutinas y los estilos de planificación que solo demuestran ventajas y 
desventajas, pues lo que ha dominado durante muchos años en la gestión institucional, en estos casos, es la fórmula invariable de planificar y de promover las soluciones que dejan a los Centros Educativos después de mucho tiempo en un poco más de lo mismo.

\section{Propuesta de una visión compartida}

La propuesta de Senge en este sentido es sobre la necesidad de construir una visión que debe estar en función de un marcado liderazgo, el cual propone conjuntamente una imagen de futuro que se procura alcanzar en un período de tiempo establecido, y que a su vez es el resultado de la participación de todos los miembros de una organización determinada en la construcción de éste.

No se podrá concebir a una organización exitosa y por ende inteligente y pensante, sin metas, valores y visiones que sean compatibles con la organización, por lo tanto, el asunto no es solamente una responsabilidad de un director o directora, si no que tales aspectos deben unir a las personas en función de una aspiración en común, en consecuencia, la educación costarricense debe mirar con prioridad la integración de planes estratégicos que partan de realidades previamente estudiadas, en las que se propicie el aporte individual y colectivo de todos los miembros de la organización escolar.

\section{Aprendizaje en equipo}

Este tema no se puede considerar que es del todo nuevo en la administración ni en la vida social. Caben muchas inquietudes sobre el trabajo en equipo, y lo más importante es sobre el aprendizaje en grupo, y es aquí donde podemos identificar una de las premisas que Senge ha dado sobre su planteamiento organizacional y es precisamente que la inteligencia del equipo supera la inteligencia de sus integrantes y cuando sus equipos crecen en el desarrollo de sus funciones no solo son capaces de generar resultados extraordinarios, si no que, sus integrantes crecen con mayor rapidez. El aprendizaje en equipo debe tener en los Centros Educativos como soporte principal el diálogo y la capacidad para encontrar posiciones consensuales. Esto significa que tanto los comités, departamentos y las áreas que están bajo la coordinación específica de un docente deben tener una predisposición para encarar los factores críticos como experiencias que sustentan su aprendizaje y su desarrollo profesional, para ello, es necesario la eliminación de los focos de tensión, discrepancias y las posiciones intransigentes que en la mayoría de los casos han sometido a los Centros Educativos a un letargo sin precedente, que los hace verse anodinos ante los cambios beligerantes que experimenta la sociedad capitalista.

\section{Dominio personal}

Se asume como aquella habilidad que se tiene para incidir en personas o cosas. Para Senge, la gente con alto dominio personal es capaz de alcanzar coherentemente los resultados que más le interesan. Abordan la vida como un artista abordaría la creación de una obra de arte, lo consigue además, integrándose a un aprendizaje inquietante, entendiéndose esto último como la facultad de integrar las experiencias individuales y sociales dentro de una misma proyección que permite lograr las metas prefijadas.

Así mismo, este autor define este rango como una disciplina que permite aclarar y ahondar continuamente nuestra visión personal que finalmente, implica concentrar nuestras energías en ver la realidad objetivamente. Esta es quizás, una de las características más deseables que deben poseerse en el liderazgo del Sistema Educativo que ante los hechos de la vida nacional se tienen los discursos 
elocuentes cargados de adulaciones y a menudo sin importancia para la comunidad educativa. Se trata por lo tanto, de un liderazgo que debe demostrar madurez, ecuanimidad y sensatez en el tratamiento de los temas que son importantes para todos los miembros de la organización, lo que significa tratar de llegar a una visión diáfana y llena de lucidez sobre el acontecer educativo.

\section{Pensamiento sistémico}

Esta es la disciplina que integra a las otras, uniéndolas en un cuerpo coherente de teoría y práctica, impidiéndoles que se tengan como actitudes separadas, sin una orientación sistemática. Al hacer un énfasis en las demás disciplinas el pensamiento sistémico, nos recuerda que, en efecto, el todo puede superar la suma de las partes; por lo tanto, Senge recomienda, que antes de aplicar las disciplinas, deben estudiarse los problemas de la organización con mayor profundidad, por lo que a menudo pasa inadvertido en medio de una barahunda de los hechos cotidianos. Además, es necesario mirar más hallá de los hechos cotidianos de las personalidades, de los acontecimientos y de esta forma examinar las estructuras que modelan los actos individuales y crear las condiciones que posibilitan cierto tipo de acondicionamiento.

Siendo el pensamiento sistémico el marco en el cual descanza toda su propuesta, Senge manifiesta: “...Denomino el pensamiento sistémico la quinta disciplina porque es la piedra angular de las cinco disciplinas de aprendizaje puestas en este libro. Todas se relacionan con su campo de enfoque: ver totalidades en vez de partes, y en vez de ver a las personas como seres que se limitan a reaccionar con impotencia, las vemos como participantes activos en la modelación de la realidad, en vez de reaccionar ante el presente, crea el futuro. Sin pensamiento sistémico no hay motivos ni medios para integrar las cinco disciplinas de aprendizaje una vez que se introducen en la práctica”.
Es aquí donde los problemas de la deserción, disciplina, bajo rendimiento, la falta de actualización en las tareas pedagógicas, factores que afectan las relaciones humanas, elaboración de adecuaciones curriculares y, en general la planificación, demandan de un abordaje coherente que obedezca a una alternativa de solución integral. Por tanto, los miembros de la organización escolar, al considerar el tratamiento de cada uno de estos temas, deben necesariamente abandonar sus esquemas conceptuales, demostrar su capacidad para afrontar con sabiduría y verdadera disposición el aprendizaje y el trabajo en equipo, que permitan llevar a la práctica soluciones que dejan de ser efímeras para ser seculares.

Hasta aquí se ha tratado de focalizar los distintos procesos que tienen lugar en la administración escolar, con la intención de profundizar el análisis y llamar la atención sobre aquellas cuestiones que fuera de ser cotidianas transcurren sin que se les preste la suficiente atención, por ende no debe olvidarse que la credibilidad y legitimación del papel que juegan los Centros Educativos en nuestra sociedad no es para siempre, más aún debe ser ganado en todos los terrenos del quehacer educativo, sobre todo en una época en la que la calidad de la educación pública una y otra vez ha sido cuestionada.

Conviene resaltar que la escuela es una organización sumamente compleja, puesto que realizar un análisis sobre uno de sus aspectos implica afectar su ambiente social, es decir, los niveles de interacción, motivación entre otros factores que no hacen posible en poco tiempo los cambios que son deseables para su mejoramiento.

Un abordaje de la escuela en su complejidad significa asumir que todas sus dimensiones conforman de manera conjunta interdependiente su vida, la cual está destinada a promover el aprendizaje y el conocimiento, a fin de tener una educación de calidad, que pueda satisfacer las necesidades individuales de todo ser humano. 
Se han hecho todas estas observaciones porque se considera que la forma en cómo se organiza una Institución Educativa, sin duda afecta su planificación, su funcionamiento, la capacidad de rendimiento, la administración, el servicio y su relación con todas las fuerzas vivas de la comunidad que además afectan su imagen pública y sus posibilidades de desarrollo, haciéndose acreedora de las críticas del medio, por no dar flexibilidad al cambio que le dé actualidad y progreso en el logro de sus objetivos institucionales, provocando de esta forma un mejor desempeño en el contexto social que le circunda.

\section{Referencias bibliográficas}

Chiavenato, Idalberto. "Introducción a la teoría general de administración". 2 ed. México: Mc Graw Hill. 1998.

Crozier, Michael. "El cambio en las organizaciones públicas”. En: Gestión Política Pública. Vol. I., No. 1. México. 1992. Págs. 93-100.

Hermida, Jorge; Serra, Roberto; Kastica, Eduardo. "Administración Estratégica y Práctica”. Buenos Aires. Ediciones Macchi. 1992.

Ivan Illich. "Un mundo sin Escuelas". México. Editorial Nueva Imagen. 1979.

Meza Spitta, Leonor. "La administración educativa en América Latina: el caso de Honduras". 1999.

Paulo Freire. "Pedagogía del oprimido". Siglo Veintiuno Editores. México. 1987.

Radetich, Horacio. "Educación, conocimiento y saber". En: Foro Universitario. Revista del Sindicato de Trabajadores de la Universidad Autónoma de México. No. 30. México. 1983.

Santos Guerra, Miguel Ángel. "Cultura y Poder para la organización escolar" . Barcelona. Editorial Siglo XXI. 1992.

Senge, Peter M. "La Quinta Disciplina". Ediciones Juan Granica, S. A. Beltrán 107, bajos 2a. 08023. Barcelona, España.

Randolf Aguirre Aguirre Asistente del Director del Departamento de Educación Especial del Ministerio de Educación Pública 